\title{
ACUTE LUPUS MYOCARDITIS TREATED WITH INTRAVENOUS IMMUNE GLOBULIN (IVIG) DUE TO CONCURRENT PULMONARY TUBERCULOSIS: A CASE REPORT
}

Andre Lucas Ribeiro ${ }^{1, \star}$, André Lucas Ribeiro ${ }^{1}$, Odirlei Andre Monticielo ${ }^{1}$, Natalia Sarzi Sartori ${ }^{1}$, Andrese Aline Gasparin ${ }^{1}$, Afonso Guilherme Schmidt ${ }^{1}$, Andrea Worm Furtado ${ }^{1}$, Augusto Emilio Hinterholz ${ }^{1}$, Larissa Martinelli Dullius ${ }^{1}$, Larissa Vargas Cruz $^{1}$

1.Hospital de Clínicas de Porto Alegre, Porto Alegre (RS), Brazil.

*Corresponding author: lucasribeiro.andre@gmail.com

\section{BACKGROUND}

Systemic lupus erythematosus (SLE) is a chronic autoimmune disease that can present with variable clinical features affecting almost every organ system. Its cardiac involvement can range from an asymptomatic pericardial effusion to cardiogenic shock due to severe myocarditis. Lupus myocarditis has a prevalence of 8 to $25 \%$, with a higher risk in the African-American ethnicity. We report a case of SLE acute myocarditis treated with intravenous immune globulin (IVIG) as an alternative protocol due to concurrent pulmonary tuberculosis precluding the use of intravenous steroid pulse therapy.

\section{CASE REPORT}

A 19-year-old, black, female patient, with SLE characterized by serositis, severe class II lupus nephritis, and antiphospholipid syndrome (APS) presented to the emergency department with elevated liver enzymes induced by antituberculosis drugs (i.e., RHZE), and restarted a few weeks before for pulmonary tuberculosis. On admission, the patient was using prednisone $1 \mathrm{mg} / \mathrm{kg}$ and tuberculosis treatment was switched for levofloxacin, amikacin, and ethambutol. After two weeks, the patient developed sudden dyspnea with signs of distributive shock. Initial investigation ruled out septic shock and other infections besides tuberculosis. A transthoracic echocardiogram revealed cardiogenic shock with global biventricular dysfunction, a reduced ejection fraction of $19 \%$ and a tricuspid annular plane systolic excursion (TAPSE) of $9 \mathrm{~mm}$. No previous heart conditions were known and a normal echocardiogram was performed 10 months before. The new dilated cardiomyopathy was attributed to SLE after multidisciplinary discussion with the ICU and Cardiology teams. Due to concurrent pulmonary tuberculosis, the myocarditis was treated with methylprednisolone (MP) $1 \mathrm{mg} /$ $\mathrm{kg}$ combined with IVIG $1 \mathrm{~g} / \mathrm{kg}$ on two consecutive days. The patient responded well to therapy and a repeat echocardiogram was performed 11 days later, showing complete myocardial recovery with an ejection fraction of $65 \%$ and a TAPSE of $19 \mathrm{~mm}$. The patient continued MP $1 \mathrm{mg} / \mathrm{kg}$ and, two months later, started mycophenolate mofetil (MMF). Currently, four months later, the patient is on remission while taking MP $0.5 \mathrm{mg} / \mathrm{kg}$ and MMF $1 \mathrm{~g}$ BID whilst treating the pulmonary tuberculosis.

\section{CONCLUSION}

Systemic lupus erythematosus patients often present with an infection triggering a disease flare. This common scenario presents the recurrent dilemma of increasing immunosuppression in a context of a severe infection. Pulse therapy, in addition to cyclophosphamide, is considered first-line therapy for myocarditis. Here we presented a successful case of lupus myocarditis treated with IVIG, which is an alternative therapy that can be safely used amidst a severe infection.

\section{KEYWORDS}

Lupus myocarditis, Immune globulin, Tuberculosis. 